\title{
Controlling the charge state of single Mo dopants in a $\mathrm{CaO}$ film
}

\author{
Yi Cui, ${ }^{1}$ Niklas Nilius, ${ }^{1,2, *}$ Hans-Joachim Freund, ${ }^{1}$ Stefano Prada, ${ }^{3}$ Livia Giordano,,${ }^{3, *}$ and Gianfranco Pacchioni ${ }^{3}$ \\ ${ }^{1}$ Fritz-Haber-Institut der Max-Planck-Gesellschaft, Faradayweg 4-6, 14195 Berlin, Germany \\ ${ }^{2}$ Carl von Ossietzky Universität Oldenburg, Institut für Physik, D-26111 Oldenburg, Germany \\ ${ }^{3}$ Dipartimento di Scienza dei Materiali, Università di Milano-Bicocca, via Cozzi 53, 20125 Milano, Italy
}

(Received 5 July 2013; revised manuscript received 29 October 2013; published 21 November 2013)

\begin{abstract}
Recent experiments have demonstrated that tiny amounts of Mo impurities give rise to drastic changes in the adsorption characteristic of a wide-gap $\mathrm{CaO}(001)$ film. In this scanning tunneling microscopy (STM) and density functional theory paper, we elucidate the underlying mechanism by analyzing the energy levels of the Mo dopants as a function of their oxidation state and depth below the surface. We show that $\mathrm{Mo}^{2+}$ ions in $\mathrm{CaO}$ subsurface layers can be reversibly charged and discharged by inducing local band-bending effects with the STM tip. A similar charge switching is not possible for Mo species in a higher oxidation state, as their highest-occupied molecular orbitals are located well below the onset of the $\mathrm{CaO}$ conduction band. The easiness of charge switching in $\mathrm{Mo}^{2+}$ ions explains the remarkable chemical properties of doped $\mathrm{CaO}$ films, as it renders the material a strong electron donor to adsorbates bound to the oxide surface.
\end{abstract}

DOI: 10.1103/PhysRevB.88.205421

PACS number(s): 73.20.Hb, 68.37.Ef, 68.55.Ln, 73.61.Ng

\section{INTRODUCTION}

Doping has proved to be a powerful approach to tailor the properties of gap materials and forms the basis for fundamental applications in microelectronics, optics, and heterogeneous catalysis. The underlying principles are well established for semiconductors, ${ }^{1}$ where doping levels down to $10^{15} \mathrm{~cm}^{-3}$ can be safely installed. In contrast, the concept of doping is less developed for oxides, as various peculiarities of these materials hamper detailed control over the dopants' characteristics. Oxides are subject to self-doping with either native defects or unknown impurities, the concentration of which is difficult to adjust experimentally. ${ }^{2}$ Both lattice defects and external impurities may adopt different charge states in the oxide lattice, ${ }^{3}$ a variability that leads to strong compensation effects and is less common in semiconductors. Moreover, dopants in wide-gap oxides are often electrically inactive, as thermal excitation is insufficient to lift the electrons from their defect states in the gap to the bulk bands. As a result, the excess charges remain bound to their host ions.

This situation may change if adsorbates with low-lying affinity levels bind to the surface and charge transfer between dopants and adspecies becomes possible. ${ }^{4,5}$ In a recent example, we showed that electron donation from Mo dopants in a $\mathrm{CaO}$ matrix substantially increases the adhesion of gold and leads to the formation of negatively charged adislands of monolayer (ML) height. ${ }^{6}$ In addition, Mo dopants were found to promote the dissociation of $\mathrm{O}_{2}$ on the $\mathrm{CaO}$ surface, a process of pivotal importance for many oxidation reactions. ${ }^{7}$ These examples underline the impact of doping in heterogeneous catalysis and should motivate concerted research activities on how to tailor the physical and chemical properties of wide-gap oxides by means of doping. ${ }^{8}$

The nature of dopants in oxide materials has been comprehensively studied by theoretical means, ${ }^{9-12}$ while experimental verification of the derived concepts is still in its infancy. One obvious obstacle is the insulating character of wide-gap oxides that renders application of electron-mediated analytical tools difficult and calls for optical and magnetic techniques. ${ }^{13,14}$ Their main disadvantage is the averaging character of the experiments that ignores local influences on the dopant properties, such as the interplay with other impurities or the surface. In contrast, spatially resolved approaches are suited to probe the distribution of dopants in the lattice and to determine the critical length over which charge transfer processes are active. Resolving these issues is key to understand the role of dopants in the surface chemistry of oxides. So far, most spatially resolved experiments along this line have been performed on semiconducting oxides, in particular on $\mathrm{TiO}_{2}$ (Refs. 15 and 16) and $\mathrm{ZnO} .{ }^{17}$ Given their relatively small band gaps, however, these materials are improper models to mimic the wide-gap insulating oxides typically used in catalysis.

In this paper, we employ scanning tunneling microscopy (STM) at $10 \mathrm{~K}$ and density functional theory (DFT) to investigate the spatial distribution and electronic properties of individual Mo dopants in a $\mathrm{CaO}$ film with 7.7-eV band gap. We show that near-surface dopants are electrically active and can be charged and discharged in the electric field of the STM tip. This flexibility results from the proximity of the highest-occupied molecular orbital (HOMO) of the Mo-donor to the onset of the oxide conduction band (CB).

\section{EXPERIMENTS}

$\mathrm{CaO}$ films of 25-ML thickness have been prepared by physical vapor deposition of $\mathrm{Ca}$ onto a sputtered and annealed Mo(001) surface in $5 \times 10^{-7}$ mbar of oxygen. ${ }^{18}$ Crystallization of the films was achieved by vacuum annealing to 800-1100 $\mathrm{K}$. The procedure resulted in a sharp $(1 \times 1)$ square pattern in low-energy electron diffraction (LEED), indicating the formation of a crystalline $\mathrm{CaO}$ surface with (001) termination. The corresponding STM images displayed atomically flat oxide terraces, being separated by a network of step edges and dislocation lines that run along the nonpolar $\mathrm{CaO}[100]$ directions (Fig. 1(a)). STM imaging and spectroscopy was possible only for bias voltages above the $\mathrm{CB}$ onset that was found to vary between 2.0 and $4.0 \mathrm{~V}$ depending on the thickness and doping level of the film. Applying negative or low-positive bias caused the tip to crash into the surface, suggesting that our films were too thick for electron transport via tunneling. In order to stabilize the tip on top of the 


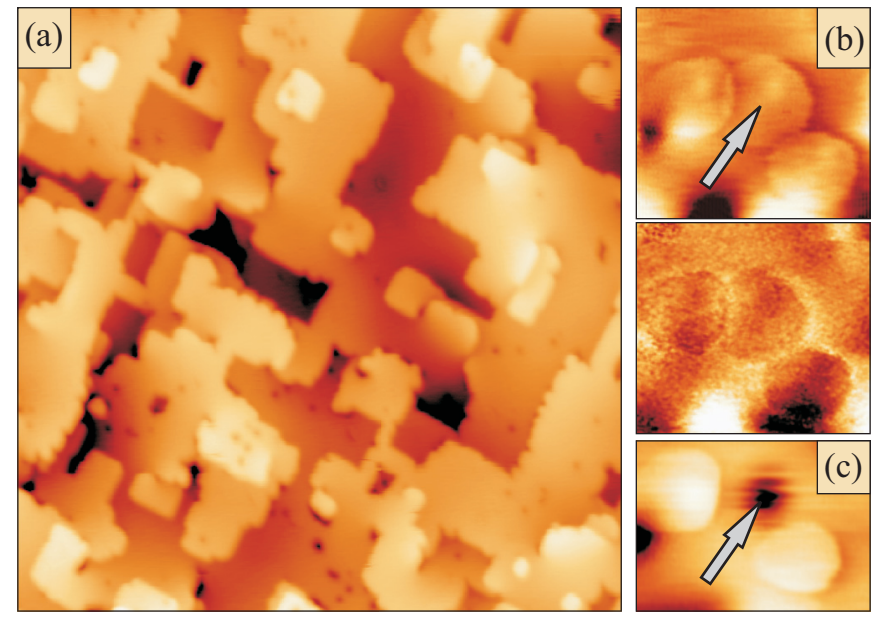

FIG. 1. (Color online) (a) Overview STM image of 25-ML CaO grown on $\mathrm{Mo}(001)\left(50 \times 50 \mathrm{~nm}^{2}, 4.5 \mathrm{~V}\right)$. (b) Topographic and $d I / d V$ image of charging rings induced by three Mo donors in the surface region. (c) Adsorption of an $\mathrm{O}_{2}$ molecule (dark minimum) onto the central feature that leads to a suppression of the charging ring $\left(11 \times 8 \mathrm{~nm}^{2}, 2.5 \mathrm{~V}\right)$.

insulating film, we could only use tunnel currents below 25 $\mathrm{pA}$ in our experiments.

\section{RESULTS AND DISCUSSION}

Earlier experiments indicated that our $\mathrm{CaO}$ films are selfdoped due to spontaneous Mo diffusion out of the support at high temperature. ${ }^{18}$ Directly at the metal-oxide interface, the extra Mo occupies $25 \%$ of the cationic sites in the rocksalt lattice and arranges in a $2 \times 2$ superstructure, as confirmed by LEED. With increasing film thickness, the Mo concentration decreases due to the large activation energy for Mo diffusion, until hardly any dopants are detectable in the near-surface region of a 25-ML film. For even thicker films, Mo needs to be artificially introduced either via ion implantation or excessive heating of the sample to $1100 \mathrm{~K} .{ }^{6}$ The presence of the Mo dopants strongly suppresses the formation of $\mathrm{O}$ vacancies in the lattice, because both defect types are potential electron donors and give rise to an increasing charge imbalance in the oxide film. The compensating defects for donor-type Mo ions would be cationic $\left(\mathrm{Ca}^{2+}\right)$ vacancies with pronounced acceptor character, which are indeed observed in the experiment. Up to now, the presence of Mo ions in the lattice could be confirmed only with nonlocal techniques, such as Auger spectroscopy, or indirectly from the modified adsorption behavior of doped with respect to pristine $\mathrm{CaO}$ films. ${ }^{4,7}$ With the following data, we are able to determine position and charge state of the dopants in a direct and spatially resolved experiment.

High-bias STM images show a smooth morphology of the film, only perturbed by a few point and line defects. With decreasing bias, characteristic rings appear on the $\mathrm{CaO}$ surface, the diameter of which varies with the tunneling conditions (Fig. 1(b)). Whereas in topographic images the rings are discernible on the basis of their enhanced height as compared to the surrounding surface, a bright circumference appears in differential conductance $(d I / d V)$ maps taken with

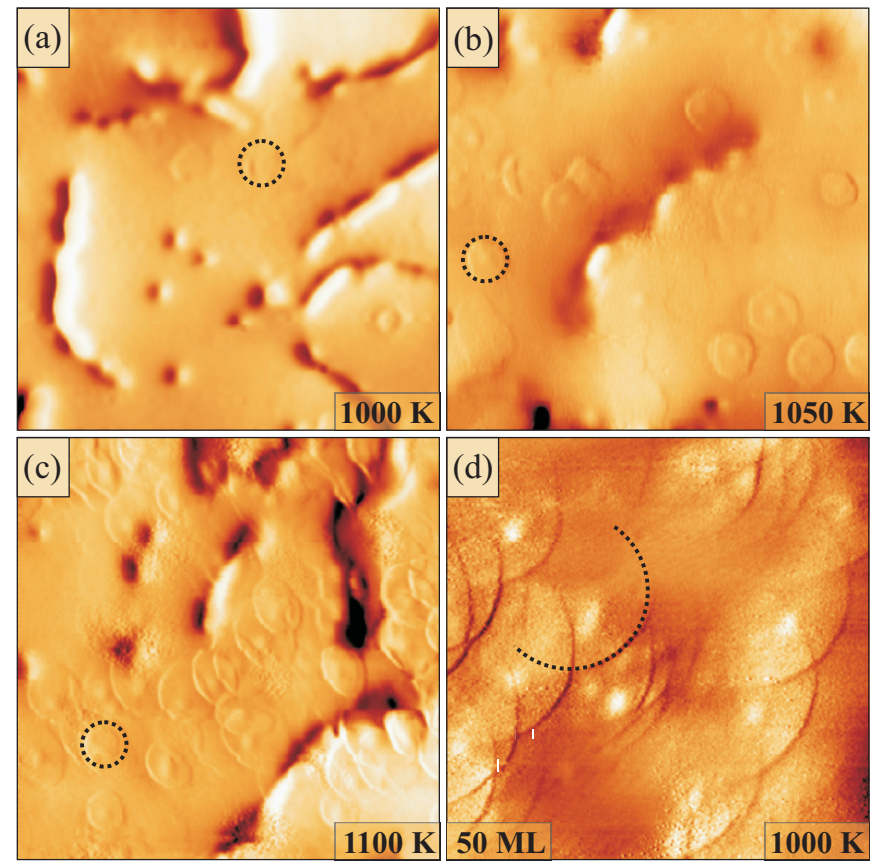

FIG. 2. (Color online) (a)-(c) STM images of 25-ML CaO annealed to the given temperatures $\left(30 \times 30 \mathrm{~nm}^{2}, 2.6 \mathrm{~V}\right)$. Note the increasing number of charging rings (exemplified by the broken circle) upon annealing. (d) On 50-ML-thick films, the diameter of charging rings is larger due to the inferior dielectric screening $\left(30 \times 30 \mathrm{~nm}^{2}, 4.4 \mathrm{~V}\right)$.

the lock-in technique. In both cases, the surface plane is perfect, and no atom-size defects are identified in the ring centers. To connect the ring features in STM with Mo dopants in the lattice, we have changed the Mo concentration by annealing the films to different temperatures, thus altering the thermodynamic stimulus for Mo diffusion (Fig. 2). The density of ring structures was found to rise from $5 \times 10^{11}$ to $5 \times 10^{12}$ $\mathrm{cm}^{-2}$ when going from low- to high-temperature films. In the latter case, the rings overlapped without visible interference, suggesting that the observed features originated from isolated species in the oxide matrix.

The rings seen in STM can be explained with a reversible charging and discharging of the Mo dopants in the tip-electric field, a mechanism that has been identified for impurity ions in semiconducting ${ }^{17,19}$ and organic layers. ${ }^{20}$ In detail, the Mo ions located at $\mathrm{Ca}$ substitutional sites introduce several discrete states in the $\mathrm{CaO}$ band gap that are of $\mathrm{Mo} 4 d$ character. ${ }^{5}$ While their energy is mainly governed by the octahedral crystal field exerted by neighboring $\mathrm{O}^{2-}$ anions, their occupancy reflects the oxidation state of the dopants. Charging may occur if a positively biased tip induces a local upward bending of the $\mathrm{CaO}$ bands, resulting in a destabilization of the dopant energy levels with respect to the unperturbed regions away from the tip. ${ }^{17,21}$ As a consequence, an electron might be transferred out of the Mo-HOMO state into one of the two possible final states (Fig. 3(a)). For dopants close to the interface, the HOMO might be pushed above the Fermi level of the system and electron transfer occurs into empty states of the metal substrate. In contrast, the HOMO electron of a near-surface dopant might be promoted into the $\mathrm{CB}$ of a neighboring $\mathrm{CaO}$ region that is 

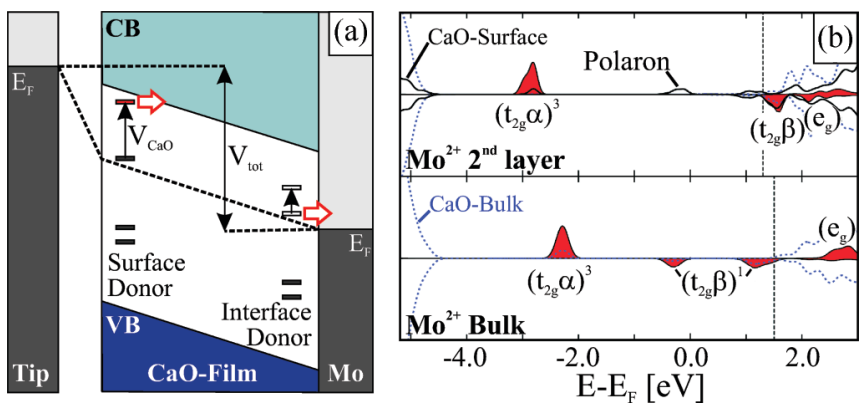

FIG. 3. (Color online) (a) Potential diagram of a STM junction containing a doped $\mathrm{CaO}$ film. The tip-electric field induces an upward bending of the oxide bands, which leads eventually to an electron transfer from the $\mathrm{Mo}^{2+} \mathrm{HOMO}$ to the $\mathrm{CB}$ of an unperturbed $\mathrm{CaO}$ region nearby. (b) Projected density of states of a $\mathrm{Mo}^{2+}$ ion located in the second subsurface plane, as well as in the $\mathrm{CaO}$ bulk. The $\mathrm{CB}$ onset is marked by a dashed vertical line; Mo-induced states are shown in red.

less affected by the tip-induced band bending and therefore lower in energy.

The amount of band bending in STM can now be given by the bias ratio between the voltage drop inside the oxide due to insufficient dielectric screening $V_{\mathrm{CaO}}$ and the total applied bias $V_{\text {tot }}: \frac{V_{\text {tot }}}{V_{\mathrm{CaO}}}=\left(\frac{\varepsilon_{\mathrm{CaO}} d_{\mathrm{Vac}}}{\varepsilon_{\mathrm{vac}} d_{\mathrm{CaO}}}+1\right){ }^{22}$ Here, $\varepsilon_{\mathrm{CaO}}=10$ is the oxide dielectric constant, while $d_{\mathrm{vac}}$ and $d_{\mathrm{CaO}}$ denote the widths of the vacuum gap $(\sim 1 \mathrm{~nm}$ at $4.0 \mathrm{~V})$ and the oxide film $(6 \mathrm{~nm})$, respectively. In the bias window in which charging rings have been observed in STM $(4.0-6.0 \mathrm{~V})$, the tip-induced band bending amounts to $1.5-2.5 \mathrm{~V}$ in near-surface $\mathrm{CaO}$ planes but reduces to $0.5 \mathrm{~V}$ in the vicinity of the metal-oxide interface.

A first hint on the more likely of the two charging routes can be obtained by comparing these numbers with the position of the Mo defect levels, ${ }^{23}$ as computed with a Perdew-Burke-Ernzerhof hybrid functional-DFT approach, as implemented in VASP. ${ }^{24,25}$ In the ideal $\mathrm{CaO}$ bulk lattice with a computed band gap of $6.1 \mathrm{eV}$, a Mo ${ }^{2+}$ substitutional ion has an electron configuration of $\left(t_{2 g} \alpha\right)^{3}\left(t_{2 g} \beta\right)^{1} e_{g}{ }^{0}$, with the $t_{2 g} \beta$-type HOMO located $1.8 \mathrm{eV}$ below the CB onset (Fig. 3(b)). When approaching the surface, the band gap experiences a gradual reduction due to the drop of the Madelung potential. As a consequence, the $\mathrm{Mo}^{2+} \mathrm{HOMO}$ effectively shifts toward the band edge and finally merges with the CB by forming a polaron with strong $4 s$ character. Formally, the $\mathrm{Mo}^{2+}$ loses one electron to the surface polaron, which in turn becomes stabilized due to the attractive potential of the resulting $\mathrm{Mo}^{3+}$ ion. The new polaronic state is spatially localized (small polaron), in agreement with its low energy with respect to the $\mathrm{CB}$ edge (1.6 eV for $\mathrm{Mo}^{2+}$ in the second subsurface plane). In contrast, a $\mathrm{Mo}^{2+}$ directly in the surface would retain all four $4 d$ electrons and, due to the $C_{4 V}$ symmetry, assume a high-spin $(e)^{2}\left(b_{2}\right)^{1}\left(a_{1}\right)^{1}\left(b_{1}\right)^{0}$ configuration with the HOMO at $3.5 \mathrm{eV}$ below the $\mathrm{CB}$ edge. Also in bulk $\mathrm{CaO}, \mathrm{Mo}^{3+}$ ions may form via a spontaneous charge transfer from the impurity to an electrontrapping defect, e.g., a dislocation line. ${ }^{4}$ However, these ions are insensitive to charge switching with the STM tip, as their $\left(t_{2 g} \alpha\right)$-type HOMO lies almost $5 \mathrm{eV}$ below the CB edge.

The same arguments hold for dopants in the interface region, which spontaneously lose one electron to the metal substrate and adopt a stable $3+$ charge state (Fig. 3(a)). The remaining $\left(t_{2 g} \alpha\right)^{3}$ electrons cannot be removed by the STM tip anymore, as the required energy to reach the metal Fermi level $(1.5 \mathrm{eV})$ is much larger than the maximum band bending in the interface region of the oxide $(0.5 \mathrm{eV})$. Charging is generally excluded for Mo ions in higher oxidation states (i.e., $+4,+5$, and +6 ), as their electrons occupy low-lying states in the $\mathrm{CaO}$ gap that cannot be destabilized by a tip-induced perturbation. In conclusion, from all species considered here, only the $\mathrm{Mo}^{2+}$ ions in the vicinity of the surface are susceptible to charge switching in the tip-electric field. The reason is that the energy distance between the Mo-HOMO and the CB edge of the unperturbed oxide matches the magnitude of band bending only for those species.

This theoretical conclusion can be corroborated by a direct experimental test. After dosing oxygen, as an electron-accepting molecule, the ring structures disappear from the STM images (Fig. 1(b)-1(d)). Apparently, the HOMO electron is no longer available for charge transfer processes triggered by the tip, as it was trapped already by the low-lying $2 \pi^{*}$ level of the $\mathrm{O}_{2}$ molecule. As electron transport through the $\mathrm{CaO}$ matrix is expected to be limited to typical tunneling distances $(1 \mathrm{~nm})$, we take this result as additional evidence for the localization of the active Mo dopants in a near-surface region of the oxide. More importantly, the disappearance of the charging rings in the presence of $\mathrm{O}_{2}$ adspecies provides an immediate connection between the tip-induced charge switching of the Mo ions and the specific redox properties of the doped $\mathrm{CaO}$ films, as discussed in Refs. 6 and 7.

Final support for our charging scenario of Mo ions in the tip-electric field comes from a careful analysis of the observed ring diameters in STM and their dependence on the tunneling parameters (Fig. 4). The ring structures become

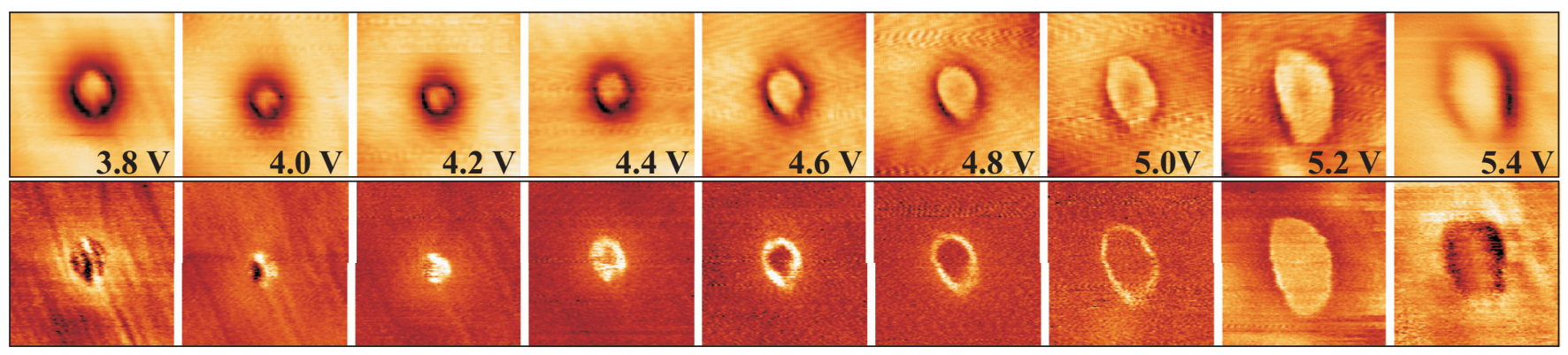

FIG. 4. (Color online) Topographic (top row) and $d I / d V$ (bottom row) maps showing the charging rings associated to a single Mo donor in $\mathrm{CaO}(001)$ measured as function of bias voltage. The asymmetry of the ring reflects the deviation of the tip apex from a perfect sphere $\left(5 \times 5 \mathrm{~nm}^{2}\right)$. 

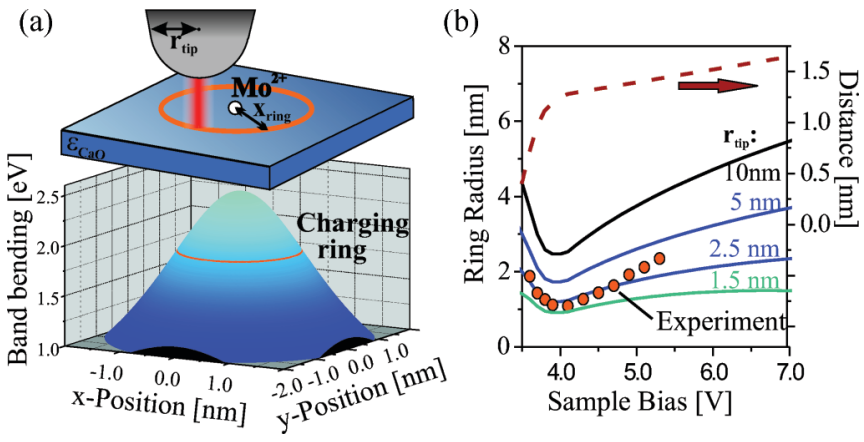

FIG. 5. (Color online) (a) Real-space model of a charging ring and shift of the $\mathrm{Mo}^{2+} \mathrm{HOMO}$ calculated for a 5.0-V sample bias and a 2.5-nm tip radius. Electron transfer into the $\mathrm{CB}$ takes place when the level crosses the orange line $(\Delta E=1.6 \mathrm{eV})$. (b) Ring diameter shown as a function of tip radius and sample bias. The experimental tip-sample distances that enter the calculations are marked by the dashed line.

visible due a certain nonlocality of band-bending effects in the STM, which enables a charge transfer out of a Mo donor even at tip positions away from the dopant. ${ }^{21}$ Mathematically, charging occurs when the tip-induced band offset at the dopant position $V_{\mathrm{CaO}}(x)$ matches the binding energy of the Mo HOMO with respect to the unperturbed $\mathrm{CB}$ edge (Fig. 5(a)). At this moment, the bound electron delocalizes over an extended $\mathrm{CaO}$ region and the positive charge at the donor site increases. The associated Coulomb attraction partially compensates for the tip-induced band shift. As a result, the tip electrons find more final states for tunneling and a circular region around the donor appears bright. Simultaneously, a sharp ring pops up in the conductance maps due to the sudden rise of the accessible $\mathrm{CaO}$ state density after charging (Fig. 1(b)). The deviation of the perturbed region from an ideal circle thereby mirrors the asymmetry of the tip apex that in turn produces radial differences in the band bending.

By considering the impact of the tip-electric field, not only at the tip apex but also at a given distance, the radius of charging rings $x_{\text {ring }}$ can be calculated from the bias division in the tunnel junction as before: $\frac{V_{\text {tot }}}{V_{\mathrm{CaO}}(x)}=\frac{\varepsilon_{\mathrm{CaO}}\left(d_{\mathrm{vac}}+r_{\mathrm{tip}}-\sqrt{r_{\text {tip }}^{2}-x_{\text {ring }}^{2}}\right)}{\varepsilon_{\mathrm{vac}} d_{\mathrm{CaO}}}+1$. The ring appears in the STM data when the band-bending $V_{\mathrm{CaO}}$ at lateral distance $x_{\text {ring }}$ matches the energy gap between the Mo HOMO and the CB onset. For the following calculations, the gap has been set to $1.6 \mathrm{eV}$ according to a $\mathrm{Mo}^{2+}$ ion in the second subsurface plane, while the tip-sample distance $d_{\text {vac }}$ was determined from experimental $z-V$ curves by adding a distance offset of $0.5 \mathrm{~nm}$. Figure 5(b) shows the computed ring radii as a function of sample bias and tip radius $r_{\text {tip }}$. Apparently, the model reproduces the general trend observed in the experiment. Initially, the ring size decreases with bias voltage as the tip rapidly retracts from the surface when the oxide $\mathrm{CB}$ becomes accessible for tunneling. At higher bias, the band bending becomes the dominant factor and the ring size increases again (Fig. 4). Reasonable agreement between simulation and experiment is obtained for an effective tip radius of $2.5 \mathrm{~nm}$, a value that is compatible with the spatial resolution in our images.

Further support for our model comes from the detection of larger ring sizes on thicker oxide films, as apparent in Fig. 2(c) and 2(d) measured on 25- and 50-ML-thick films, respectively. Thicker films have an inferior screening ability, which increases the band-bending effects in the tip-electric field and thus the observed ring sizes. In contrast, all charging rings in one image series have roughly the same size, which indicates a similar depth of the active Mo dopants below the surface. In the case of a random distribution of Mo ions with respect to the surface, we would expect a larger distribution of ring diameters than observed in our experiment.

\section{CONCLUSIONS}

Characteristic ring features on the surface of a $\mathrm{CaO}$ film have been connected to reversible charge switches of Mo impurities in the tip-electric field of an STM junction. The rings appear as local band-bending effects lift the Mo eigenstates above the $\mathrm{CB}$ onset of unperturbed oxide regions away from the tip, causing the Mo ions to become temporary ionized. We have shown that only $\mathrm{Mo}^{2+}$ ions in near-surface regions of the oxide are potentially suited for such charge transfer processes; the HOMOs of higher-oxidized species are located too far below the oxide $\mathrm{CB}$. The direct observation of charging effects of Mo dopants suggests a similar scenario to be responsible for the unusual chemical response of Mo-doped $\mathrm{CaO}$ films. ${ }^{4,6,7}$ Whereas in the present paper tip-induced band bending provides the empty states for the electron drain out of the Mo ions, the respective affinity levels were introduced by electron-accepting adsorbates, such as gold and oxygen, in earlier work. In both cases, the behavior of the Mo ions is compatible with that of an electron donor. The clarification of the underlying charge transfer processes may help us in designing the properties of doped oxide materials in the future.

\section{ACKNOWLEDGMENTS}

This paper was supported by the Excellence Initiative 'UNICAT' of the German Science Foundation and the Italian Basic Research Investment Fund (FIRB) Project No. RBAP115AYN. We also thank the European COST Action No. CM1104. Y.C. is grateful for a fellowship from the Humboldt Foundation.

\footnotetext{
*Corresponding authors: nilius@fhi-berlin.mpg.de; livia.giordano@ mater.unimib.it

${ }^{1}$ B. I. Shklovskii and A. L. Efros, Electronic Properties of Doped Semiconductors (Springer, Berlin, Germany, 1984).
}

${ }^{2}$ S. Wendt et al., Science 320, 1755 (2008).

${ }^{3}$ H. Y. Kim, H. M. Lee, R. G. S. Pala, V. Shapovalov, and H. Metiu, J. Phys. Chem. C 112, 12398 (2008); V. Shapovalov and H. Metiu,

J. Catal. 245, 205 (2007). 
${ }^{4}$ F. Stavale et al., J. Am. Chem. Soc. 134, 11380 (2012).

${ }_{5}^{5}$ S. Prada, L. Giordano, and G. Pacchioni, J. Phys. Chem. C 117, 9943 (2013).

${ }^{6}$ X. Shao, S. Prada, L. Giordano, G. Pacchioni, N. Nilius, and H.-J. Freund, Angew. Chem. Int. Ed. 50, 11525 (2011).

${ }^{7}$ Y. Cui, X. Shao, M. Baldofski, J. Sauer, N. Nilius, and H.-J. Freund, Angew. Chem. Int. Ed. 125, 11595 (2013).

${ }^{8}$ X. Y. Sun, B. Li, and H. Metiu, J. Phys. Chem. C 117, 7114 (2013).

${ }^{9}$ C. G. Van de Walle, Phys. Rev. Lett. 85, 1012 (2000).

${ }^{10}$ M. Nolan and G. W. Watson, Surf. Sci. 586, 25 (2005).

${ }^{11}$ G. Pacchioni et al., J. Phys. Condens. Matter 16, S2497 (2004).

${ }^{12}$ J. Robertson and S. J. Clark, Phys. Rev. B 83, 075205 (2011).

${ }^{13}$ A. Fujishima, X. Zhang, and D. A. Tryk, Surf. Sci. Rep. 63, 515 (2008); T. Umebayashi, T. Yamaki, H. Itoh, and K. Asai, Appl. Phys. Lett. 81, 454 (2002).

${ }^{14}$ P. Myrach et al., ChemCatChem 2, 854 (2010).

${ }^{15}$ M. Batzill, K. Katsiev, D. J. Gaspar, and U. Diebold, Phys. Rev. B 66, 235401 (2002).

${ }^{16}$ A. Sasahara and M. Tomitori, J. Phys. Chem. C 117, 17680 (2013).
${ }^{17}$ H. Zheng, A. Weismann, and R. Berndt, Phys. Rev. Lett. 108, 076801 (2012); 110, 226101 (2013).

${ }^{18}$ X. Shao, P. Myrach, N. Nilius, and H.-J. Freund, J. Phys. Chem. C 115, 8784 (2011).

${ }^{19}$ K. Teichmann, M. Wenderoth, S. Loth, R. G. Ulbrich, J. K. Garleff, A. P. Wijnheijmer, and P. M. Koenraad, Phys. Rev. Lett. 101, 076103 (2008).

${ }^{20}$ G. V. Nazin, X. H. Qiu, and W. Ho, Phys. Rev. Lett. 95, 166103 (2005).

${ }^{21}$ R. M. Feenstra, Y. Dong, M. P. Semtsiv, and W. T. Masselink, Nanotechnology 18, 044015 (2007).

${ }^{22}$ S. W. Wu, G. V. Nazin, X. Chen, X. H. Qiu, and W. Ho, Phys. Rev. Lett. 93, 236802 (2004)

${ }^{23}$ In the calculations, bulk Mo ions are placed in a $2 \times 2 \times 2 \mathrm{CaO}$ supercell, while near-surface dopants are modeled with a $3 \times 3$ surface cell of a five-layer slab. For reciprocal space sampling, $3 \times 3 \times 3$ and $3 \times 3 \times 1$ Monkhorst-Pack meshes are used, respectively.

${ }^{24}$ C. Adamo and V. Barone, J. Chem. Phys. 110, 6158 (1999).

${ }^{25}$ G. Kresse and J. Hafner, Phys. Rev. B 47, 558 (1993); G. Kresse and J. Furthmüller, ibid. 54, 11169 (1996). 